\title{
Asian women have greater abdominal and visceral adiposity than Caucasian women with similar body mass index
}

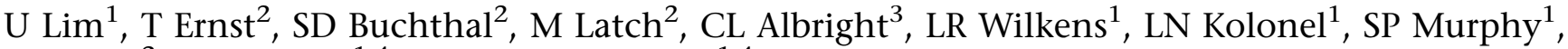 \\ L Chang $^{2}$, R Novotny ${ }^{1,4}$ and L Le Marchand ${ }^{1,4}$
}

${ }^{1}$ Epidemiology Program, University of Hawaii Cancer Center, Honolulu, HI, USA; ${ }^{2}$ Department of Medicine, John A Burns School of Medicine, University of Hawaii, Honolulu, HI, USA and ${ }^{3}$ Prevention \& Control Program, University of Hawaii Cancer Center, Honolulu, HI, USA

Background: In the Multiethnic Cohort Study, Japanese Americans (JA) have lower mean body mass index (BMI) compared with Caucasians, but show a higher waist-to-hip ratio at similar BMI values and a greater risk of diabetes and obesity-associated cancers.

Objective: We investigated the abdominal, visceral and hepatic fat distribution in these Asian and Caucasian Americans. Design: A cross-sectional sample of 60 female cohort participants (30 JA and 30 Caucasians), of ages 60-65 years and BMls $18.5-40 \mathrm{~kg} \mathrm{~m}^{-2}$, underwent anthropometric measurements and a whole-body dual energy X-ray absorptiometry (DXA) scan: a subset of 48 women also had abdominal magnetic resonance imaging (MRI).

Results: By design, JA women had similar BMls (mean $26.5 \mathrm{~kg} \mathrm{~m}^{-2}$ ) to Caucasian women $\left(27.1 \mathrm{~kg} \mathrm{~m}^{-2}\right.$ ). JA women were found to have a significantly smaller hip circumference $(96.9$ vs $103.6 \mathrm{~cm} ; P=0.007)$ but not a significantly lower DXA total fat mass (25.5 vs $28.8 \mathrm{~kg} ; P=0.16)$. After adjusting for age and DXA total fat mass, JA women had a greater waist-to-hip ratio (0.97 vs $0.89 ; P<0.0001)$, DXA trunk fat $(15.4$ vs $13.9 \mathrm{~kg} ; P=0.0004)$ and MRI \% abdominal visceral fat $(23.9 \mathrm{vs} 18.5 \% ; P=0.01)$ and a lower DXA leg fat mass (8.2 vs $10.0 \mathrm{~kg} ; P=<.0001)$. Their MRI \% subcutaneous fat $(33.4$ vs $30.2 \% ; P=0.21)$ and \% liver fat (5.8 vs $3.8 \% ; P=0.06$ ) did not significantly differ from that of Caucasian women.

Conclusions: Our findings build on limited past evidence, suggesting that Asian women carry greater abdominal and visceral fat when compared with Caucasian women with similar overall adiposity. This may contribute to their elevated metabolic risk for obesity-related diseases.

Nutrition and Diabetes (2011) 1, e6; doi:10.1038/nutd.2011.2; published online 9 May 2011

Keywords: body composition; central obesity; liver fat; race/ethnicity; subcutaneous adipose tissue; visceral adipose tissue

\section{Introduction}

Body mass index (BMI: weight $(\mathrm{kg}) /$ height $\left.(\mathrm{m})^{2}\right)$ is the most commonly used measure of overall body fatness. However, body fat deposition in the abdominal region is known to be a better predictor than BMI of the risks of cardiovascular disease, ${ }^{1}$ diabetes $^{2}$ and, possibly, obesity-associated cancers. ${ }^{3-5}$ Similarly, specific metabolic phenotypes, such as of insulin resistance, are better predicted by waist $\operatorname{size}^{6}$ and by direct measurement of trunk fat using dual energy X-ray absorptio-

Correspondence: Dr U Lim, Epidemiology Program, University of Hawaii Cancer Center, 1236 Lauhala Street, Suite 407, Honolulu, HI 96813, USA. E-mail: ulim@cc.hawaii.edu

${ }^{4}$ These authors contributed equally to this work.

Received 3 January 2011; revised 8 March 2011; accepted 20 March 2011 metry (DXA) than by BMI. $^{7}$ Studies of more detailed abdominal fat distribution using magnetic resonance imaging (MRI) or computed tomography have associated intraperitoneal visceral adipose tissue (VAT), more than subcutaneous adipose tissue (SAT), with a greater metabolic risk. ${ }^{8,9}$ This is thought to be due to the drainage of free fatty acids from VAT to the portal venous system, which, therefore, promotes hepatic insulin resistance and dyslipidemia more effectively than SAT. ${ }^{10-12}$ Thus, direct measurements of liver fat content may also be informative when investigating the metabolic effects of excess weight. It is estimated that as many as $20-30 \%$ of adults in developed countries have obesity-related nonalcoholic fatty liver disease. ${ }^{13}$

Along with age and sex, ethnicity is a major determinant of body fat distribution. Studies to date suggest that, compared with Whites, abdominal and visceral adiposity is 
greater among Latinos, and visceral adiposity is lower among African Americans. ${ }^{14-16}$ However, data comparing Asians with Caucasians are limited, although Asians manifest a higher obesity-related disease risk. ${ }^{17-19}$ In a small study, greater VAT in proportion to total adiposity was detected among young women (but not men) of mixed Asian ancestry $(n=18)$, compared with Whites $(n=36) .{ }^{20}$ In another study, greater VAT was observed in Filipinas compared with their Caucasian or African-American counterparts of similar or higher BMI. ${ }^{21}$ Greater visceral adiposity was also reported among the Canadians of Chinese descent, compared with Caucasians. ${ }^{22}$ There is an even greater paucity of data on ethnic differences in liver fat content. ${ }^{23}$ One study compared middle-aged men in Japan and non-Hispanic White men in the US and found a lower mean BMI but higher liver fat content among the Japanese. ${ }^{24}$

In an ongoing multiethnic cohort of older adults, based in Hawaii and California, we recently observed a higher BMI-adjusted waist-to-hip ratio and higher BMI-associated risks of incident diabetes ${ }^{25}$ and breast and colorectal cancers (White et al., personal communication, 2010) among Japanese Americans (JA) compared with Whites, even after adjusting for other risk factors. In order to better understand whether these risk differences may stem from different body fat distribution, we conducted detailed anthropometry, DXA and MRI studies in a subset of 60 JA and White, apparently healthy women from the same cohort. Women were chosen for this initial study because they have greater variability in waist size for a given level of BMI compared with men ${ }^{26}$ and because of their susceptibility to accumulate more abdominal adiposity after menopause. ${ }^{27}$

\section{Subjects and methods}

\section{Recruitment}

Participants for the current study were recruited from the ongoing Multiethnic Cohort Study. This prospective study conducted in Hawaii and Los Angeles consists of over 215000 men and women who were of ages $45-75$ years at baseline (1993-1996) and of mainly five racial/ethnic groups, as described previously. ${ }^{28}$

Women who were 60-65 years of age, as of September 2009, who reported both parents being of either Japanese or White ethnicity on the baseline questionnaire and who responded to the most recent Multiethnic Cohort follow-up questionnaire (completed in 2003-2008) were eligible for the present study. In order to ensure that Japanese and White women would have a similar BMI distribution, stratified sampling was performed. Equal numbers of women were recruited from the following BMI categories within each ethnic/racial group: 18.5-21.9, 22-24.9, 25-26.9, 27-29.9 and $30-35 \mathrm{~kg} \mathrm{~m}^{-2}$. The BMI category used for subject selection was determined from self-reported weight and height in the most recently completed third Multiethnic
Cohort questionnaire and confirmed during recruitment. Exclusion criteria also included: current or recent smoking ( $<2$ years); use of medications that may interfere with metabolism and body composition (chemotherapy, insulin or weight-loss drugs); substantial weight change (loss or gain of 20 pounds or more) in the past 6 months; pre- or perimenopausal status (had menses in the past 12 months); BMI outside the target range $\left(18.5-35 \mathrm{~kg} \mathrm{~m}^{-2}\right)$; and report of any soft or metal implants/objects in the body that could bias the body composition estimates or put subjects at risk in the MRI magnetic field (for example, pacemakers, cardiac valves, stents).

Out of the 218 women contacted, 46 (21\%) were ineligible because of medication use $(n=21)$, implants or mastectomy $(n=6)$, self-reported BMI $<18.5$ or $>35 \mathrm{~kg} \mathrm{~m}^{-2}(n=7)$, smoking $(n=5)$, possible claustrophobia $(n=4)$ and difficulty donating blood $(n=3)$. In addition, some women were either not reachable $(n=21$ or $10 \%)$ or unwilling to participate ( $n=77$ or $35 \%$ ). Among the 74 remaining willing and eligible women (34\%), we selected 60 women (30 Japanese, 30 Whites) to obtain a similar distribution of BMI in the two ethnic groups.

Participants underwent anthropometric measurements, a whole-body DXA scan and a fasting venous blood collection at the University of Hawaii Clinical Research Center and completed a brief self-administrated questionnaire on current use of dietary supplements and medications. Out of the 60 women, 48 also agreed to participate in an abdominal MRI scan at the University of Hawaii and Queen's Medical Center MR Research Center. The Institutional Review Boards of University of Hawaii and Queen's Medical Center approved the study protocol, and all participants signed an informed consent.

\section{DXA and anthropometric measurements}

Participants first visited the University of Hawaii Clinical Research Center for a series of examinations. A whole-body DXA scan (GE Lunar Prodigy, Madison, WI, USA) was performed to measure total and regional body fat mass in the trunk, arms and legs, as well as lean soft tissue mass and bone mineral content, ${ }^{29}$ from which percent total fat (total fat mass divided by total body mass) was derived. The trunkto-periphery fat mass ratio was calculated as an indicator of abdominal fat distribution by dividing the fat mass in the trunk by the sum of fat mass in the arms and legs. A certified radiographic technician operated the DXA according to a standard protocol. Calibration using a manufacturer's phantom was performed daily.

Anthropometric measurements included standing and sitting heights, weight, waist and hip circumferences, biacromial and bi-iliac breadths and chest depth. Leg length was obtained by subtracting sitting height from standing height, and proportional leg length (or leg-to-height ratio) by dividing leg length by height. Waist circumference was taken at two locations, that is, at the navel and immediately 
above the iliac crest. ${ }^{30}$ Hip circumference was measured at the widest area between waist and thighs, including buttocks. The breadth and depth measurements were obtained using a Lafayette Caliper. ${ }^{31}$ Each measurement was taken twice, and a third time if the two measures differed by $>0.1 \mathrm{~kg}$ or by $>0.5 \mathrm{~cm}$, after which the average of the two closest values was used in the analysis.

\section{Magnetic resonance imaging scan}

Procedure and equipment. In total, $20 \mathrm{JA}$ and 28 White women completed a one-time MRI scan within 1 to 13 weeks after the DXA scan on a research-dedicated 3 Tesla TIM Trio scanner (Siemens Medical Systems, Ehrlangen, Germany; software version VB13), using the body radio frequency coil for excitation and a six-element body matrix coil in conjunction with six elements from the multichannel spine coil for signal detection. The women were scanned in a supine position for approximately $30 \mathrm{~min}$, with their abdominal area covered with the body matrix coil. Participants were instructed to hold their breath for 10-17 s during the gradient echo sequences in order to minimize respiratory motion artifacts.

First, sagittal and coronal localizer scans were performed to identify the liver and the specific lumbar spine position (L4-L5) of interest. Next, a series of axial triple-gradient-echo scans, using a fast low-angle shot (FLASH) sequence, was performed on the liver with the following set-up: $10 \times 6 \mathrm{~mm}$ slices, $40 \mathrm{~cm}$ field of view, $256 \times 256$ resolution, time to echo $(\mathrm{TE})=2.4,3.7$ and $5.0 \mathrm{~ms}$, time to relaxation $(\mathrm{TR})=160 \mathrm{~ms}$, $25^{\circ}$ flip angle, one average with radiofrequency spoiling and generalized autocalibrating partially parallel acquisition $($ GRAPPA $)=2$. The time to echo values were optimized to acquire water and lipid signals in-phase, out of phase and again in-phase. ${ }^{32-34}$ An additional set of lipid-only gradient-echo images with water suppression was obtained. Acquisition parameters were $35 \mathrm{~cm}$ field of view, $30 \times 5 \mathrm{~mm}$ slices, TR/TE/TD (or difference in echo times) $=140 / 2.6$ / $6000 \mathrm{~ms}, 70^{\circ}$ flip angle, one average, two concatenations, $320 \times 256$ resolution, generalized autocalibrating partially parallel acquisition $=2$, with two $80 \mathrm{~mm}$ suppression slabs with a $20 \mathrm{~mm}$ gap, parallel to the slices. All scans were immediately inspected for the presence of motion artifacts and repeated if any were visible.

Estimation of visceral/subcutaneous fat. Analyses were performed off-line using the National Institutes of Health program, Image J (http://rsbweb.nih.gov/ij). Each subject's cross-sectional water-suppressed image at L4-L5 spine position was segmented to a black and white image (with thresholding) using the binary function to emphasize the fat borders. The abdominal wall was traced manually first, and the total fat area (in $\mathrm{mm}^{2}$ ) was determined (Figure 1a). Next, the peritoneal wall was traced manually, and the visceral fat area (intraperitoneal, including fat inside internal organs) was determined, which was then subtracted from the first measurement to obtain the subcutaneous fat area (extraperitoneal). Percent visceral and subcutaneous fat was then calculated by dividing each area by the abdominal area.

Estimation of percent liver fat. Using a Siemens Leonardo workstation, a circular region of interest $\left(15-25 \mathrm{~cm}^{2}\right)$ was defined in the lateral portion of the right lobe of the liver on the triple-echo fast low-angle shot images; care was taken to avoid the large vessels (Figure 1b). The signal intensities were determined for all three echo times. The signal intensities of the first and third echoes were averaged, yielding $\mathrm{S}^{+}$(signal of water and lipid in-phase), and the signal intensity of the

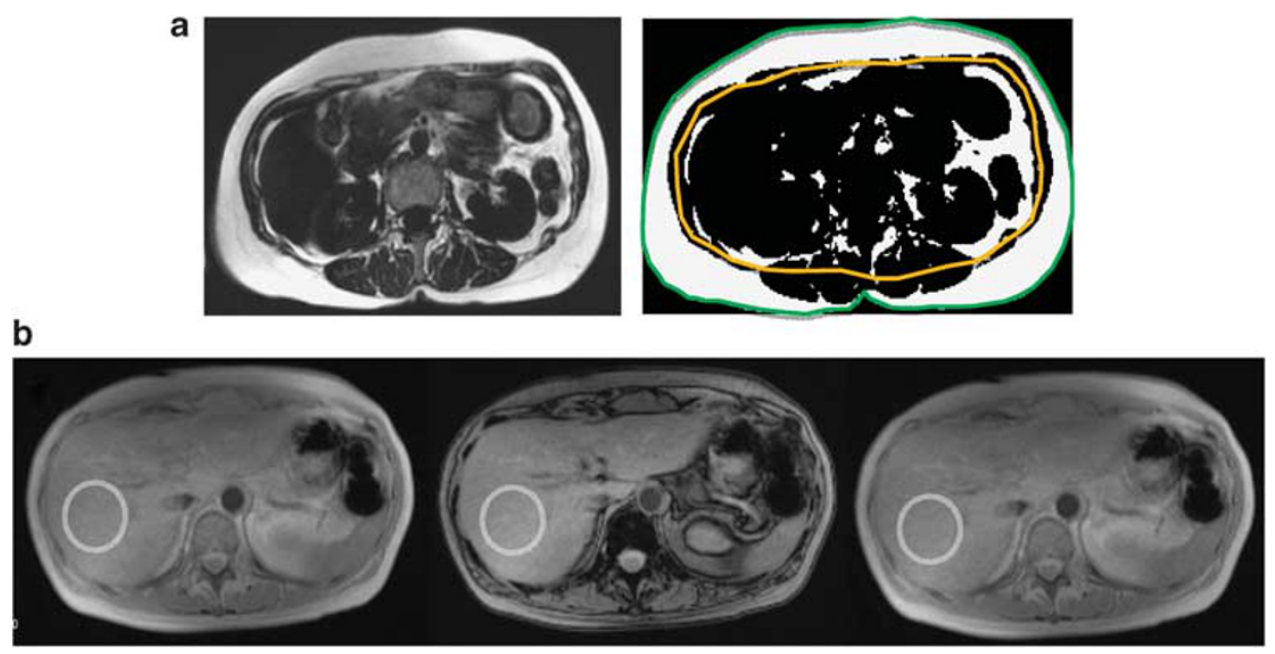

Figure 1 Abdominal fat distribution assessed by magnetic resonance imaging. (a) Visceral and subcutaneous fat distribution at L4-L5: water-suppressed image (left) and binarized image with tracings for total cross-sectional area (green) and peritoneal membrane (yellow; right), differentiating the abdominal fat tissue (white) into the visceral component inside the peritoneum and the subcutaneous fat outside. (b) Liver fat assessment: abdominal images were obtained at three echo times $-2.4 \mathrm{~ms}$ (in-phase, left), $3.7 \mathrm{~ms}$ (out of phase, center) and $5.0 \mathrm{~ms}$ (in-phase, right), from which average image intensity for liver fat estimation was measured in the right lobe of the liver (region of interest marked in circles), avoiding visible vessels. 
second echo yielded $\mathrm{S}^{-}$(signal of water and lipid out of phase). From these signal intensities, the relative fat content of the liver was calculated as follows:

$$
F=\left(S^{+}-S^{-}\right) /\left(1.7 S^{+}-0.3 S^{-}\right)
$$

where the water content of the liver is assumed to be $70 \%{ }^{34}$

\section{Statistical analysis}

All analyses were performed using the SAS Statistical Software, version 9.2 (SAS Institute, Inc., Cary, NC, USA). Demographic and lifestyle characteristics were compared between JA and White women using Wilcoxon rank-sum test for continuous variables or using the $\chi^{2}$-test of association for categorical variables. The main analysis included ethnic comparisons of indicators for total or overall adiposity (BMI and DXA total fat mass), central or abdominal adiposity (waist circumference, waist-to-hip ratio, DXA trunk fat mass and trunk-to-periphery fat ratio) and peripheral adiposity (DXA leg fat mass and arm mass). In addition, abdominal fat distribution into visceral vs subcutaneous fat components was assessed using the amount of MRI visceral and subcutaneous fat area at L4-L5 as percentages of MRI total abdominal area. Also, hepatic adiposity (MRI percent liver fat) was compared. Mean adiposity values were compared between JA and White women using analysis of covariance. The model assumptions were met for all models, although log-transformation was required for MRI percent liver fat.
Models were adjusted for age, or age and DXA total fat mass. DXA total fat mass was adjusted for because the study objective was to compare abdominal, visceral and hepatic adiposity at similar levels of overall adiposity. Because JA women had a significantly lower leg-to-height ratio and lower DXA leg fat mass, models were additionally adjusted for these variables to explore whether peripheral fat storage, indicated by these measures, explained the ethnic differences in abdominal, visceral and hepatic adiposity. Ethnic comparison of adiposity indicators was further stratified by three BMI categories (normal weight, overweight and obese), and heterogeneity across BMI categories was examined by including a cross-product term of ethnicity and BMI variables in the analysis of covariance model for each adiposity indicator, in which significance was determined by the $P$-value from the Wald test of the product term. A nominal level of $\alpha=0.05$ was used for statistical significance. In addition, $P$-values corrected for multiple comparisons by computing false discovery rate, as shown below, are presented. ${ }^{35} P_{\mathrm{FDR}}=\min \left(P_{(\mathrm{i}+1)},(m / i) P_{(i)}\right)$ (where $P_{(i)}$ is the ranked $P$-value and $\mathrm{m}$ is the number of tests)

\section{Results}

By study design, mean age and BMI values were similar for the JA and White participants (Table 1). The upper range of BMI computed from measured weight and height was

Table 1 Characteristics ${ }^{\mathrm{a}}$ of Japanese American and White female participants

\begin{tabular}{|c|c|c|c|}
\hline & Japanese American $(\mathrm{N}=30)$ & White $(\mathrm{N}=30)$ & P-value \\
\hline Age & $63.6(60.9,65.7)$ & $63.6(61.2,65.8)$ & 0.87 \\
\hline Weight, kg & $62.2(44.9,88.0)$ & $69.2(50.5,100.8)$ & 0.08 \\
\hline Height, cm & $154(143,165)$ & $161(147,167)$ & $<0.0001$ \\
\hline Leg-to-height ratio & $0.45(0.44,0.49)$ & $0.47(0.44,0.50)$ & $<0.0001$ \\
\hline Body mass index (BMI), $\mathrm{kg} \mathrm{m}^{-2}$ & $26.5(19.0-35.2)$ & $27.1(18.8-39.6)$ & 0.83 \\
\hline Waist circumference at navel, $\mathrm{cm}$ & $94.4(70.3,123.4)$ & $93.5(71.5,134.9)$ & 0.81 \\
\hline Waist circumference at iliac crest, $\mathrm{cm}$ & $96.1(78.0,125.3)$ & $96.9(78.4,131.9)$ & 0.54 \\
\hline Hip circumference, $\mathrm{cm}$ & $96.9(84.7,124.5)$ & $103.6(88.7,130.8)$ & 0.007 \\
\hline Bi-acromial breadth, $\mathrm{cm}$ & $37.0(34.2,38.8)$ & $36.7(32.8,39.0)$ & 0.88 \\
\hline Bi-iliac breadth, cm & $30.8(28.0,38.9)$ & $31.1(27.8,37.6)$ & 0.44 \\
\hline Chest depth, cm & $18.7(15.6,25.5)$ & $20.0(17.2,27.2)$ & 0.02 \\
\hline Hormone treatment, \% current use & $1(3)$ & $6(20)$ & 0.04 \\
\hline Dietary supplement, \% current use & $26(87)$ & $25(83)$ & 0.72 \\
\hline BMI difference since cohort entry in $1993-1996^{\mathrm{b}}$ & $1.9(-3.1,5.4)$ & $3.1(-3.4,11.3)$ & 0.18 \\
\hline Education, years ${ }^{\mathrm{b}}$ & $14(12,18)$ & $16(12,18)$ & 0.04 \\
\hline Smoking history, $n(\%)^{\mathrm{b}}$ & & & 0.06 \\
\hline Never & $22(73)$ & $15(50)$ & \\
\hline Former & $8(27)$ & $15(50)$ & \\
\hline \multicolumn{4}{|l|}{ Diet } \\
\hline$\%$ Calories from fat ${ }^{b}$ & $30(20,44)$ & $30(12,44)$ & 0.94 \\
\hline$\%$ Calories from carbohydrates ${ }^{\mathrm{b}}$ & $55(38,67)$ & $54(38,74)$ & 0.29 \\
\hline Physical activity ${ }^{\mathrm{b}}$, metabolic equivalents (MET) & $1.57(1.01,2.14)$ & $1.61(1.19,2.17)$ & 0.17 \\
\hline
\end{tabular}


somewhat higher than the one based on self-report during recruitment $\left(18.5-35 \mathrm{~kg} \mathrm{~m}^{-2}\right)$. Compared with White women, JA women tended to have a shorter standing height, lower leg-to-height ratio, lower hip circumference and a smaller chest depth. There was no significant difference between the two groups in weight, waist circumference or bi-acromial and bi-iliac breadths. JA women were also less likely than Whites to be currently using hormone replacement therapy, but were equally likely to be taking dietary supplements. Information available from the Multiethnic Cohort baseline questionnaire indicated that JA women had slightly fewer years of education and were less likely to be a former smoker than White women, but showed similar BMI gain since cohort entry, similar baseline dietary intake of fat or carbohydrate as percent calories and similar baseline physical activity (Table 1). The subset of women within each ethnic group who had undergone the MRI scan (20 JA and 28 White women) did not differ significantly from those who did not have an MRI, with regard to mean BMI, leg-to-height ratio, waist-to-hip ratio or DXA total and trunk fat mass (data not shown).

Table 2 compares the mean adiposity measures between JA and White women. All comparisons were adjusted for age, which provided essentially the same results as the unadjusted estimates in this study. Adiposity measures were further adjusted for DXA total fat mass (last column in Table 2) to compare fat distribution between the two ethnic groups at similar levels of overall adiposity. DXA total fat was significantly positively correlated with all the regional adiposity indicators.

Compared with White women, and despite similar BMI and DXA total or percent fat mass, JA women had a significantly greater mean waist-to-hip ratio, measured either at the navel or immediately above the iliac crest, due to their lower hip circumference (Table 2). This difference remained statistically significant after adjustment for DXA total fat mass. Ageadjusted DXA trunk fat mass was similar in JA and White women but was greater in JA women after adjustment for total fat mass $(P=0.0004)$. DXA leg fat mass was markedly smaller $(P=0.0007)$ and trunk-to-periphery fat ratio markedly greater $(P=0.006)$ in JA women compared with White women. These ethnic differences remained statistically significant after adjusting for total fat mass.

MRI measures were not significantly different between JA and White women in the subset of women who underwent this examination, although mean percent visceral fat and percent liver fat tended to be greater $(P=0.09)$ in JA than in White women (Table 2$)$. The difference in percent visceral fat reached statistical significance $(P=0.01)$ after further adjusting for total fat mass, and the difference in percent liver fat

Table 2 Comparison ${ }^{a}$ of overall, abdominal, visceral and hepatic adiposity in Japanese American and White women

\begin{tabular}{|c|c|c|c|c|c|c|c|}
\hline & \multicolumn{3}{|c|}{$\begin{array}{l}\text { Age-adjusted mean } \\
\text { (95\% confidence limit) }\end{array}$} & \multicolumn{4}{|c|}{$\begin{array}{c}\text { Age- and DXA total fat mass-adjusted mean } \\
\text { (95\% confidence limit) }\end{array}$} \\
\hline & Japanese American & White & P-value & Japanese American & White & P-value & P-FDR \\
\hline \multicolumn{8}{|l|}{ Total adiposity } \\
\hline Body mass index (BMI) & $26.5(24.7,28.3)$ & $27.0(25.1,28.8)$ & 0.72 & $27.3(26.8,27.9)$ & $26.1(25.5,26.7)$ & 0.003 & 0.006 \\
\hline DXA total fat mass, $\mathrm{kg}$ & $25.5(22.2,28.8)$ & $28.8(25.5,32.1)$ & 0.16 & - & - & - & - \\
\hline DXA \% total fat & $40.1(37.5,42.7)$ & $41.0(38.5,43.6)$ & 0.60 & - & - & - & - \\
\hline \multicolumn{8}{|l|}{ Abdominal Adiposity } \\
\hline Waist circumference (at navel), $\mathrm{cm}$ & $94.7(89.4,100.1)$ & $95.1(89.7,100.4)$ & 0.93 & $97.2(95.3,99.2)$ & $92.6(90.6,94.5)$ & 0.002 & 0.005 \\
\hline Hip circumference, $\mathrm{cm}$ & $98.3(94.7,101.9)$ & $105.4(101.8,109.0)$ & 0.008 & $100.0(98.8,101.3)$ & $103.7(102.4,105.0)$ & 0.0001 & 0.0004 \\
\hline Waist-to-hip ratio (waist at navel) & $0.96(0.94,0.99)$ & $0.90(0.87,0.92)$ & 0.0008 & $0.97(0.95,0.99)$ & $0.89(0.87,0.91)$ & $<0.0001$ & 0.0005 \\
\hline Waist-to-hip ratio (waist at iliac crest) & $0.98(0.96,1.00)$ & $0.94(0.92,0.96)$ & 0.005 & $0.98(0.97,1.00)$ & $0.93(0.92,0.95)$ & $<0.0001$ & 0.0005 \\
\hline DXA trunk fat mass, $\mathrm{kg}$ & $14.3(12.2,16.5)$ & $14.9(12.8,17.1)$ & 0.68 & $15.4(14.8,15.9)$ & $13.9(13.4,14.5)$ & 0.0004 & 0.001 \\
\hline DXA trunk-to-periphery fat ratio & $1.38(1.26,1.49)$ & $1.14(1.03,1.26)$ & 0.006 & $1.41(1.30,1.51)$ & $1.11(1.01,1.22)$ & 0.0002 & 0.0007 \\
\hline \multicolumn{8}{|l|}{ Peripheral adiposity } \\
\hline DXA leg fat mass, kg & $7.7(6.7,8.8)$ & $10.4(9.4,11.5)$ & 0.0007 & $8.2(7.6,8.7)$ & $10.0(9.4,10.5)$ & $<0.0001$ & 0.0005 \\
\hline DXA arm fat mass, $\mathrm{kg}$ & $2.5(2.2,2.9)$ & $2.6(2.3,2.9)$ & 0.81 & $2.7(2.5,2.9)$ & $2.5(2.3,2.6)$ & 0.08 & 0.10 \\
\hline \multicolumn{8}{|l|}{ Abdominal fat distribution } \\
\hline MRI abdominal area at $\mathrm{L} 4-\mathrm{L} 5, \mathrm{~cm}^{2}$ & $574(473,675)$ & $646(561,732)$ & 0.27 & $601(528,674)$ & $627(565,688)$ & 0.59 & 0.64 \\
\hline MRI \% visceral fat area/abdominal area & $23.1(19.5,26.6)$ & $19.1(16.2,22.1)$ & 0.09 & $23.9 \%(20.8 \%, 27.0 \%)$ & $18.5 \%(15.9 \%, 21.1 \%)$ & 0.01 & 0.02 \\
\hline MRI \% subcutaneous fat/abdominal area & $32.3(27.9,36.8)$ & $31.0(27.2,34.8)$ & 0.65 & $33.4 \%(29.6 \%, 37.3 \%)$ & $30.2 \%(27.0 \%, 33.5 \%)$ & 0.21 & 0.25 \\
\hline MRI visceral-to-subcutaneous fat ratio & $0.76(0.53,0.99)$ & $0.74(0.55,0.94)$ & 0.90 & $0.78(0.55,1.02)$ & $0.73(0.53,0.92)$ & 0.73 & 0.73 \\
\hline \multicolumn{8}{|l|}{ Hepatic adiposity } \\
\hline MRI\% liver fat (geometric mean) & $5.7(4.1,8.0)$ & $3.9(2.9,5.2)$ & 0.09 & $5.8 \%(4.2 \%, 8.0 \%)$ & $3.8 \%(2.9 \%, 5.0 \%)$ & 0.06 & 0.08 \\
\hline MRI fatty liver $(>5.5 \%)$ & $49(28,70)$ & $26(8,44)$ & 0.10 & $51 \%(31 \%, 72 \%)$ & $24 \%(7 \%, 41 \%)$ & 0.05 & 0.08 \\
\hline
\end{tabular}

Abbreviations: DXA, dual energy X-ray absorptiometry; MRI, magnetic resonance imaging; $P$-FDR, $P$-value corrected for multiple comparisons using the false discovery rate method. ${ }^{a}$ Mean (95\% confidence limit) is presented from analysis of covariance with adjustment for either (1) age or (2) age and DXA total body fat mass. Participants included 30 Japanese American and 30 White women for anthropometry and DXA measures and 20 Japanese American and 28 White women for MRI measures. 
became of borderline significance $(P=0.06)$. Using a cut point of $5.5 \%$ liver fat for diagnosis of fatty liver, ${ }^{36,37}$ the greater prevalence of fatty liver in JA was of borderline statistical significance, compared with White women after adjusting for total fat mass $(P=0.05)$.

Results were similar when BMI instead of DXA total fat mass was used to adjust for total adiposity (data not shown): JA women showed a significantly greater waist-to-hip ratio, lower DXA leg fat, greater DXA trunk-to-periphery fat ratio and greater MRI percent visceral fat. However, in contrast to the adjustment for total fat mass, adjusting for BMI did not yield a difference in DXA trunk fat mass between JA and White women $(14.6 \mathrm{~kg}$ in JA vs $14.9 \mathrm{~kg}$ in White women; $P=0.86)$.

Adjustment for multiple comparisons did not change the significance of the findings but slightly attenuated the ethnic difference in MRI percent liver fat and fatty liver prevalence (Table 2, $P$-value corrected for multiple comparisons using the false discovery rate method, in the rightmost column).

Because JA women had a significantly lower leg-to-height ratio and lower DXA leg fat mass, adiposity analyses were further adjusted for either variable to explore if the ethnic differences are in part explained by these skeletal differences or reflect a greater tendency of White women to deposit fat in the legs. When adjusted for leg-to-height ratio, the ethnic patterns described above remained, but the differences were attenuated for MRI percent visceral fat $(22.8 \%$ in JA vs $19.4 \%$ in White women; $P=0.20)$ and for percent liver fat $(5.1 \mathrm{vs}$ $4.2 \% ; P=0.46)$. Adjusting for DXA leg fat mass attenuated most of the differences, except for waist-to-hip ratio (measured at the navel, 0.95 vs $0.91 ; P=0.0006$ ), and resulted in a significantly higher MRI percent subcutaneous fat in JA women (35.1 vs $29.0 \% ; P=0.04$ ).

\section{Discussion}

This cross-sectional study examined the body fat distribution of JA and White women of comparable BMI distribution, following the observation of a higher BMI-associated risk of diabetes and cancers among the former group in a large ongoing cohort study. JA and white women were found to have similar mean DXA total fat mass and waist circumference, but JA had a significantly smaller mean hip circumference and DXA leg fat mass. When regional adiposity indicators were adjusted for DXA total fat to compare proportional body fat distribution at similar levels of overall adiposity, greater MRI abdominal and visceral adiposity were observed among JA than in White women. JA women did not differ from Caucasians in measures of MRI abdominal subcutaneous fat and had only a nonsignificantly greater MRI percent liver fat and prevalence of fatty liver.

Our findings confirm previous studies that reported a high prevalence of central obesity among Asian ethnic groups. ${ }^{38}$ In addition, our results are consistent with limited past data that suggested a greater visceral fat deposition in young Asian-American women and in older Filipinas compared with Caucasians. ${ }^{20,21}$ VAT is a better predictor of metabolic syndrome than abdominal SAT ${ }^{9,39}$ and may explain, at least partially, the very high rates of Type 2 diabetes ${ }^{17,25}$ and colorectal cancer risk $^{18,40,41}$ among Japanese and JAs. In addition to the physical proximity and connection of VAT to the liver, VAT appears to possess unique molecular properties with higher lipolytic activity and greater secretion of proinflammatory products compared with SAT. ${ }^{42}$

We also examined percent liver fat by MRI, which has rarely been compared between Asians and non-Hispanic Whites from a single-source population. We found that JA women tended to have a higher percent liver fat and prevalence of fatty liver, compared with White women, although these differences did not reach statistical significance. This finding is consistent with an earlier study that detected a higher liver fat content among Japanese middle-aged men in Japan, compared with non-Hispanic White men in the US, despite a higher mean BMI in the latter group. ${ }^{24}$ Using the cutoff value for percent liver fat of 5.5\%, as suggested for the MRIbased diagnosis of fatty liver disease, ${ }^{36,37} 49 \%$ of JA and $26 \%$ of White participants in our study were likely to have fatty liver disease. This ethnic difference grew larger after adjusting for DXA total fat. Although we were unable to take into account the variation in fat content throughout the entire liver, our results, based on a partial assessment, further support the hypothesis that a greater tendency to accumulate hepatic fat may contribute to the higher risk of insulin resistance and dyslipidemia of JAs. ${ }^{24,43}$

It is not clear why Asian Americans, who, on the average, have a lower overall adiposity, have a tendency to develop proportionally more abdominal and visceral adiposity. It may be related to Asians' lower capacity to store fat in peripheral regions, starting from an early age. We and others have observed higher central adiposity and lower peripheral adiposity in Asian women, as early as in adolescence and young adulthood. ${ }^{31,44}$ In the present study, we found that the higher abdominal adiposity and lower leg-fat mass in JA women were not entirely explained by their lower height, smaller leg-to-height ratio or lower chest depth. Other biological characteristics common to Asian men and women may contribute to their higher central adiposity and lower peripheral adiposity.

Our findings of greater abdominal and visceral adiposity among JA than White women were consistent across measurements, based on anthropometry, DXA and MRI. Waist-to-hip ratio was particularly good at reflecting the ethnic-specific fat distribution patterns and remained significantly different between the groups, even after adjustments for DXA total fat, proportional leg length or leg fat mass. Findings were similar but somewhat attenuated for waist circumference or waist-to-height ratio, as compared with the waist-to-hip ratio. Thus, simple measures of waist and hip circumferences appear to be a good indicator of the difference in abdominal fat between older JA and White women. 
This study was limited to women, and the findings may not be generalizable to men. As one of the two previous studies in Asian Americans was also limited to women ${ }^{21}$ and the other reported greater VAT in Asian women but not in Asian men, compared with Whites, ${ }^{20}$ it would be important to also examine men and include other Asian groups than JAs.

In our MRI study, one cross-sectional scan at L4-L5 was used to estimate the VAT and SAT areas, and the estimated VAT and SAT areas served as surrogates for abdominal VAT and SAT volumes, respectively. The L4-L5 level is the most commonly used demarcation for cross-sectional MRI or computed tomography scans for total abdominal fat distribution, and these measures have shown a good correlation with volumetric measurements from 'multislice' scans. ${ }^{29,45,46}$ However, one study found that the VAT area at L2-L3 or L1-L2 was better correlated than the VAT area at L4-L5 with the total VAT volume estimated from 35 continuous computed tomography images of the abdomen (from T10-T11 to L5-S1). ${ }^{9}$ In that study, women showed less variation in VAT and SAT measured at different crosssectional levels than men. We also did not distinguish the deep vs superficial depots of the abdominal subcutaneous fat (DSAT and SSAT) - DSAT has been associated more closely with insulin resistance than SSAT or even VAT. ${ }^{47-49}$ African Americans, in particular, have been shown to have greater DSAT but less VAT than Whites, which makes the information on the sub-depots of the subcutaneous fat important for this ethnic group with relatively higher insulin resistance. ${ }^{49}$ However, in contrast to people of African ancestry, studies of Asians and Whites have shown a good correlation between VAT and metabolic risk, ${ }^{24}$ suggesting that a bias from lack of data on SAT layers is less likely.

Despite some potential limitations, this preliminary study assessed a large number of direct and indirect indicators of overall, abdominal, visceral and hepatic adiposity in understudied Asian-American women. The strong and consistent pattern that emerges of greater proportional abdominal and visceral adiposity among JA women compared with their White counterparts with similar BMI or total fat may be an important factor that contributes to their higher risk of diabetes and obesity-related cancers.

\section{Conflict of interest}

The authors declare no conflict of interest.

\section{Acknowledgements}

Funding for this study was provided in part by the University of Hawaii Cancer Center (SPM, RN, LLM), as well as by the National Cancer Institute for the Multiethnic Cohort (R37 CA54281: LNK), the National Institute of Health for the UH-QMC MR Research Center (5P20-RR11091 and G12RR003061: TE and LC) and the National Center for Research
Resources at NIH for the University of Hawaii Clinical Research Center (P20 RR11091). We thank the study participants and the dedicated staff at the University of Hawaii Cancer Center (Karin Koga, Eugene Okiyama, Naomi Hee, Janice Nako-Piburn, Janine Abe, Wileen Mau, Maj Earle), Clinical Research Center (Sara Murakami, Jane Yakuma, Patty Iwamoto) and the MR Research Center (Carol Kosaki).

\section{References}

1 Lee CM, Huxley RR, Wildman RP, Woodward M. Indices of abdominal obesity are better discriminators of cardiovascular risk factors than BMI: a meta-analysis. J Clin Epidemiol 2008; 61: 646-653.

2 Nyamdorj R, Qiao Q, Lam TH, Tuomilehto J, Ho SY, Pitkäniemi J et al. Decoda Study Group. BMI compared with central obesity indicators in relation to diabetes and hypertension in Asians. Obesity (Silver Spring) 2008; 16: 1622-1635.

3 Larsson SC, Wolk A. Obesity and colon and rectal cancer risk: a meta-analysis of prospective studies. Am J Clin Nutr 2007; 86: 556-565.

4 Lahmann PH, Hoffmann K, Allen N, van Gils CH, Khaw KT, Tehard B et al. Body size and breast cancer risk: findings from the European Prospective Investigation into Cancer And Nutrition (EPIC). Int J Cancer 2004; 111: 762-771.

5 Seidell JC. Waist circumference and waist/hip ratio in relation to all-cause mortality, cancer and sleep apnea. Eur J Clin Nutr 2010; 64: 35-41.

6 Lee S, Bacha F, Gungor N, Arslanian SA. Waist circumference is an independent predictor of insulin resistance in black and white youths. J Pediatr 2006; 148: 188-194.

7 Nelson TL, Bessesen DH, Marshall JA. Relationship of abdominal obesity measured by DXA and waist circumference with insulin sensitivity in Hispanic and non-Hispanic white individuals: the San Luis Valley Diabetes Study. Diabetes Metab Res Rev 2008; 24: 33-40.

8 Wagenknecht LE, Langefeld CD, Scherzinger AL, Norris JM, Haffner SM, Saad MF et al. Insulin sensitivity, insulin secretion, and abdominal fat: the Insulin Resistance Atherosclerosis Study (IRAS) Family Study. Diabetes 2003; 52: 2490-2496.

9 Kuk JL, Church TS, Blair SN, Ross R. Measurement site and the association between visceral and abdominal subcutaneous adipose tissue with metabolic risk in women. Obesity (Silver Spring) 2010; 18: 1336-1340.

10 Montague CT, O'Rahilly S. The perils of portliness: causes and consequences of visceral adiposity. Diabetes 2000; 49: 883-888.

11 Kabir M, Catalano KJ, Ananthnarayan S, Kim SP, Van Citters GW, Dea MK et al. Molecular evidence supporting the portal theory: a causative link between visceral adiposity and hepatic insulin resistance. Am J Physiol Endocrinol Metab 2005; 288: E454-E461.

12 Bergman RN, Kim SP, Catalano KJ, Hsu IR, Chiu JD, Kabir M et al. Why visceral fat is bad: mechanisms of the metabolic syndrome. Obesity (Silver Spring) 2006; 14(Suppl 1): 16S-19S.

13 Preiss D, Sattar N. Non-alcoholic fatty liver disease: an overview of prevalence, diagnosis, pathogenesis and treatment considerations. Clin Sci (Lond) 2008; 115: 141-150.

14 Despres JP, Couillard C, Gagnon J, Bergeron J, Leon AS, Rao DC et al. Race, visceral adipose tissue, plasma lipids, and lipoprotein lipase activity in men and women: the Health, Risk Factors, Exercise Training, and Genetics (HERITAGE) family study. Arterioscler Thromb Vasc Biol 2000; 20: 1932-1938.

15 Wu CH, Heshka S, Wang J, Pierson Jr RN, Heymsfield SB, Laferrère B et al. Truncal fat in relation to total body fat: influences of age, sex, ethnicity and fatness. Int J Obes (Lond) 2007; 31: 1384-1391. 
16 Beasley LE, Koster A, Newman AB, Javaid MK, Ferrucci L, Kritchevsky SB et al. Inflammation and race and gender differences in computerized tomography-measured adipose depots. Obesity (Silver Spring) 2009; 17: 1062-1069.

17 Chan JC, Malik V, Jia W, Kadowaki T, Yajnik CS, Yoon KH et al. Diabetes in Asia: epidemiology, risk factors, and pathophysiology. JAMA 2009; 301: 2129-2140.

18 Center MM, Jemal A, Smith RA, Ward E. Worldwide variations in colorectal cancer. CA Cancer J Clin 2009; 59: 366-378.

19 Palaniappan LP, Wong EC, Shin JJ, Fortmann SP, Lauderdale DS. Asian Americans have greater prevalence of metabolic syndrome despite lower body mass index. Int J Obes (Lond) 2011; 35: 393-400.

20 Park YW, Allison DB, Heymsfield SB, Gallagher D. Larger amounts of visceral adipose tissue in Asian Americans. Obes Res 2001; 9: 381-387.

21 Araneta MR, Barrett-Connor E. Ethnic differences in visceral adipose tissue and type 2 diabetes: Filipino, African-American, and white women. Obes Res 2005; 13: 1458-1465.

22 Lear SA, Humphries KH, Kohli S, Birmingham CL. The use of BMI and waist circumference as surrogates of body fat differs by ethnicity. Obesity (Silver Spring) 2007; 15: 2817-2824.

23 Burgert TS, Taksali SE, Dziura J, Goodman TR, Yeckel CW, Papademetris X et al. Alanine aminotransferase levels and fatty liver in childhood obesity: associations with insulin resistance, adiponectin, and visceral fat. J Clin Endocrinol Metab 2006; 91: 4287-4294.

24 Azuma K, Kadowaki T, Cetinel C, Kadota A, El-Saed A, Kadowaki S et al. Higher liver fat content among Japanese in Japan compared with non-Hispanic whites in the United States. Metabolism 2009; 58: 1200-1207.

25 Maskarinec G, Erber E, Grandinetti A, Verheus M, Oum R, Hopping $\mathrm{BN}$ et al. Diabetes incidence based on linkages with health plans: the multiethnic cohort. Diabetes 2009; 58: $1732-1738$

26 Duncan BB, Chambless LE, Schmidt MI, Szklo M, Folsom AR, Carpenter MA et al. Correlates of body fat distribution. Variation across categories of race, sex, and body mass in the atherosclerosis risk in communities study. The Atherosclerosis Risk in communities (ARIC) Study Investigators. Ann Epidemiol 1995; 5: 192-200.

27 Lovejoy JC, Champagne CM, Smith SR, de Jonge L, Xie H. Ethnic differences in dietary intakes, physical activity, and energy expenditure in middle-aged, premenopausal women: the Healthy Transitions Study. Am J Clin Nutr 2001; 74: 90-95.

28 Kolonel LN, Henderson BE, Hankin JH, Nomura AM, Wilkens LR, Pike MC et al. A multiethnic cohort in Hawaii and Los Angeles: baseline characteristics. Am J Epidemiol 2000; 151: 346-357.

29 Glickman SG, Marn CS, Supiano MA, Dengel DR. Validity and reliability of dual-energy X-ray absorptiometry for the assessment of abdominal adiposity. J Appl Physiol 2004; 97: 509-514.

30 Bosy-Westphal A, Booke CA, Blöcker T, Kossel E, Goele K, Later W et al. Measurement site for waist circumference affects its accuracy as an index of visceral and abdominal subcutaneous fat in a Caucasian population. J Nutr 2010; 140: 954-961.

31 Novotny R, Daida YG, Grove JS, Le Marchand L, Vijayadeva V. Asian adolescents have a higher trunk:peripheral fat ratio than Whites. J Nutr 2006; 136: 642-647.

32 Kotronen A, Westerbacka J, Bergholm R, Pietilainen KH, YkiJarvinen H. Liver fat in the metabolic syndrome. J Clin Endocrinol Metab 2007; 92: 3490-3497.

33 Fischer MA, Nanz D, Reiner CS, Montani M, Breitenstein S, Leschka $\mathrm{S}$ et al. Diagnostic performance and accuracy of 3-D spoiled gradient-dual-echo MRI with water- and fat-signal separation in liver-fat quantification: comparison to liver biopsy. Invest Radiol 2010; 45: 465-470.

34 Guiu B, Loffroy R, Petit JM, Aho S, Ben Salem D, Masson D et al. Mapping of liver fat with triple-echo gradient echo imaging: validation against 3.0-T proton MR spectroscopy. Eur Radiol 2009; 19: 1786-1793.

35 Yekutieli D, Benjamini Y. A resampling based false discovery rate controlling multiple test procedure. I Statist Plann Inference 1999; 82: 171-196.

36 Szczepaniak LS, Nurenberg P, Leonard D, Browning JD, Reingold JS, Grundy S et al. Magnetic resonance spectroscopy to measure hepatic triglyceride content: prevalence of hepatic steatosis in the general population. Am J Physiol Endocrinol Metab 2005; 288: E462-E468.

37 Borra RJ, Salo S, Dean K, Lautamäki R, Nuutila P, Komu M et al. Nonalcoholic fatty liver disease: rapid evaluation of liver fat content with in-phase and out-of-phase MR imaging. Radiology 2009; 250: 130-136.

38 WHO Expert Consultation. Appropriate body-mass index for Asian populations and its implications for policy and intervention strategies. Lancet 2004; 363: 157-163.

39 Demerath EW, Reed D, Rogers N, Sun SS, Lee M, Choh AC et al. Visceral adiposity and its anatomical distribution as predictors of the metabolic syndrome and cardiometabolic risk factor levels. Am J Clin Nutr 2008; 88: 1263-1271.

40 Hata M, Sakamoto K, Doneza J, Sumida K, Sugimoto K, Ishiyama $\mathrm{S}$ et al. Improvement of long-term survival of colorectal cancer in Japanese-Americans of Hawaii from 1990 to 2001. Int J Clin Oncol 2010; 15: 559-564.

41 Yamaji T, Iwasaki M, Sasazuki S, Kurahashi N, Mutoh M, Yamamoto $\mathrm{S}$ et al. Visceral fat volume and the prevalence of colorectal adenoma. Am J Epidemiol 2009; 170: 1502-1511.

42 Wilding JP. The importance of free fatty acids in the development of Type 2 diabetes. Diabet Med 2007; 24: 934-945.

43 Wulan SN, Westerterp KR, Plasqui G. Ethnic differences in body composition and the associated metabolic profile: a comparative study between Asians and Caucasians. Maturitas 2010; 65: 315-319.

44 Freedman DS, Wang J, Thornton JC, Mei Z, Pierson Jr RN, Dietz $\mathrm{WH}$ et al. Racial/ethnic differences in body fatness among children and adolescents. Obesity (Silver Spring) 2008; 16: 1105-1111.

45 Kamel EG, McNeill G, Van Wijk MC. Usefulness of anthropometry and DXA in predicting intra-abdominal fat in obese men and women. Obes Res 2000; 8: 36-42.

46 Park YW, Heymsfield SB, Gallagher D. Are dual-energy $\mathrm{X}$-ray absorptiometry regional estimates associated with visceral adipose tissue mass? Int J Obes Relat Metab Disord 2002; 26: 978-983.

47 Kelley DE, Thaete FL, Troost F, Huwe T, Goodpaster BH. Subdivisions of subcutaneous abdominal adipose tissue and insulin resistance. Am J Physiol Endocrinol Metab 2000; 278: E941-E948.

48 Smith SR, Lovejoy JC, Greenway F, Ryan D, deJonge L, de la Bretonne $\mathrm{J}$ et al. Contributions of total body fat, abdominal subcutaneous adipose tissue compartments, and visceral adipose tissue to the metabolic complications of obesity. Metabolism 2001; 50: 425-435.

49 Goedecke JH, Levitt NS, Lambert EV, Utzschneider KM, Faulenbach MV, Dave JA et al. Differential effects of abdominal adipose tissue distribution on insulin sensitivity in black and white South African women. Obesity (Silver Spring) 2009; 17: 1506-1512.

This work is licensed under the Creative Commons Attribution-NonCommercial-No Derivative Works 3.0 Unported License. To view a copy of this license, visit http://creativecommons.org/ licenses/by-nc-nd/3.0/ 